\title{
Effect of monocytes/macrophages on the early osteogenic differentiation of hBMSCs
}

\author{
R. P. Pirraco, R. L. Reis and A. P. Marques* \\ 3B's Research Group - Biomaterials, Biodegradables and Biomimetics, University of Minho, Headquarters of the European Institute of \\ Excellence on Tissue Engineering and Regenerative Medicine, AvePark, 4806-909 Taipas, Guimarães; and ICVS/3B's - PT Government \\ Associate Laboratory, Braga/Guimarães, Portugal
}

\begin{abstract}
Heterotypic cell interactions are essential for the homeostasis of bone tissue, in particular the widely studied interaction between osteoblasts and osteoclasts. Closely related with osteoclasts are monocytes/ macrophages. These have been shown to produce osteogenic factors, e.g. BMP-2, which plays a key role in bone metabolism. However, the mechanisms through which monocytes/macrophages interact with osteoblasts are still elusive. The aim of this work was to assess the influence of human peripheral blood monocytes/macrophages over the early osteogenic differentiation of human bone marrow stromal cells (hBMSCs) in the presence of dexamethasone-supplemented medium. The co-cultures were performed using porous transwells that allowed the interaction between both cell types through the production of paracrine factors. The potential effect of BMP-2 produced by monocytes/macrophages was addressed by adding an anti-BMP-2 antibody to the co-cultures. hBMSCs cultured in the presence of monocytes/macrophages had a higher proliferation rate than hBMSCs monocultures. The quantification of early osteogenic marker alkaline phosphatase (ALP) revealed higher activity of this enzyme in cells in the co-culture throughout the time of culture. Both of these effects were inhibited by adding an anti-BMP-2 antibody to the cultures. Moreover, qRT-PCR for osteocalcin and osteopontin transcripts showed overexpression of both markers. Once again, the effect of monocytes/macrophages over hBMSC osteogenic differentiation was completely inhibited in the co-cultures by blocking BMP-2. The present report confirmed that monocytes/macrophages produce BMP-2, which promotes osteogenic differentiation and proliferation of hBMSCs cumulatively to dexamethasone-supplemented medium. This potentially implies that monocyte/macrophages play a stronger role in bone homeostasis than so far supposed. Copyright () 2012 John Wiley \& Sons, Ltd.
\end{abstract}

Received 20 October 2011; Accepted 20 October 2011

Keywords osteoblasts; monocytes; macrophages; bone biology; cell interactions

\section{Introduction}

It is well established that heterotypic cellular interactions in the bone microenvironment are essential for bone homeostasis. The demonstration that cells of the osteoblastic lineage control the formation and activity of bone-resorbing cells, the osteoclasts (Rodan and Martin, 1981), is even more relevant for biologists who intend to understand bone biology and, in particular, bone remodelling and regeneration. Bone formation and bone

\footnotetext{
*Correspondence to: A. P. Marques, 3Bs Research Group Biomaterials, Biodegradables and Biomimetics, University of Minho, Headquarters of the European Institute of Excellence on Tissue Engineering and Regenerative Medicine, AvePark, Taipas 4806-909, Guimarães, Portugal. E-mail: apmarques75@gmail.com
}

resorption, essential for a normal bone turnover, are coupled processes (Parfitt, 1982; Pirraco et al., 2009) that co-exist within a delicate balance, controlled by factors such as hormones (Mundy, 1993) and other growth factors (Linkhart et al., 1996). Osteoclasts, being multinucleated and highly specialized cells derived from the haematopoietic lineage that have the function of resorbing bone, are seen as critical players in this regulatory process (Sasaki et al., 1994; Teitelbaum et al., 1995; Teitelbaum et al., 1997). Also from the haematopoietic lineage, and closely related to osteoclasts, are circulating peripheral blood monocytes, which can easily reach almost any tissue in the human body. While in physiological conditions monocytes differentiate into resident macrophages after tissue infiltration (Hume et al., 2002), upon injury or infection, monocytes are recruited to the injury site and 
differentiate into activated macrophages (Hume et al., 2002) participating in the tissue response. In the particular case of bone tissue, after acquiring an inflammatory phenotype, for instance at a fracture site, macrophages can differentiate into osteoclasts (Haynes et al., 2001).

The role of monocytes/macrophages in bone remodelling and regeneration certainly does not rely simply upon this phagocytic lineage interplay. Monocytes/macrophages are linked to the production of several cytokines and growth factors, such as tumour necrosis factor- $\alpha$ $(\mathrm{TNF} \alpha)$, transforming growth factor- $\beta$ (TGF $\beta$ ), plateletderived growth factor (PDGF), fibroblast growth factor (FGF) and others (Einhorn, 1998; Dimitriou et al., 2005). Additionally, it has been shown that macrophages produce several osteogenic factors, such as 1,25-dihydroxyvitamin D3 (Kreutz et al., 1993) and BMP-2 (Champagne et al., 2002). All these molecules have a potential effect on other cells present at the fracture healing site, e.g. mesenchymal stem cells (MSCs) and osteoblastic progenitors (Dimitriou et al., 2005). Furthermore, it is known that activated monocytes/macrophages contribute to in vitro vascular calcification by affecting the osteoblastic differentiation of calcifying vascular cells (CVCs), a subpopulation of osteoblast-like cells derived from the artery wall (Tintut et al., 2002). Recently, some studies focusing on bone-resident macrophages proposed a complex bone regulatory role for those cells (Chang et al., 2008; Raggatt et al., 2009). These studies suggested that macrophages can be key players in bone homeostasis, acting both in bone resorption, by regulating osteoclast activity, and in osteoblast mineralization, through cytokine production. However, the precise effect of monocytes/macrophages on mesenchymal progenitor cells has been subject of very few studies, which either focus on the immunomodulatory effect of the MSCs (Aggarwal and Pittenger, 2005; Jiang et al., 2005; Ryan et al., 2005) or on the osteoclastic differentiation potential of macrophages (Udagawa et al., 1990; Fujikawa et al., 1996; Quinn, 1998).

Bone morphogenetic protein-2 (BMP-2) is part of the superfamily of TGF $\beta$ and has been shown to induce bone formation in vitro and in vivo (Schmitt et al., 1999a, 1999b; Valentin-Opran et al., 2002; Bessa et al., 2008). Its potential for bone regeneration is attested by several clinical studies involving this protein (Valentin-Opran et al., 2002). Champagne et al. (2002) demonstrated, in vitro, that macrophage cell lines influence the differentiation behaviour of human MSCs by producing BMP-2, which in turn triggers osteogenic differentiation. This was an important finding, since it eventually implicates macrophages in the process of bone healing.

The objective of this study was, therefore, to assess the effect of human peripheral blood monocytes/macrophages over the osteogenic differentiation of human bone marrow stromal cells (hBMSCs), in order to deeply understand this cross-talk and to complement what was previously reported with murine and human macrophage cell lines. For that, an indirect co-culture system, using culture inserts, was set up with hBMSCs and human peripheral blood-derived monocytes/macrophages under standard osteogenic conditions in the presence of an anti-BMP-2 antibody. The use of osteogenic culture conditions intended to further explore the effect of the culture medium, and in particular of the dexamethasone supplement, over the role of monocytes/macrophages in the osteogenic differentiation of hMSCs.

\section{Materials and methods}

\subsection{Cell isolation and culture}

Human bone marrow aspirates $(n=4)$ were obtained from routine hip revision surgeries in Hospital da Prelada (Porto, Portugal), after informed consent. Buffy coats $(n=4)$ were obtained from the Portuguese Blood Institute (IPS). All samples were acquired under cooperation agreements with the 3Bs Research Group.

Mononuclear cells from human bone marrow samples were obtained after homogenization of the samples and differential centrifugation using Histopaque 1077 (Sigma, USA). The cells were then cultured at $37^{\circ} \mathrm{C}$ in a humidified atmosphere with $5 \% \mathrm{CO}_{2}$ in Dulbecco's modified Eagle's medium (DMEM; Sigma, USA), supplemented with 10\% FBS (Invitrogen, USA) and 1\% antibiotics (A/B, Invitrogen, USA). After human bone marrow stromal cells (hBMSCs) reached confluence at passage 1, 30000 cells were plated in 24-well culture plates and cultured in $600 \mu \mathrm{l}$ osteogenic medium composed of DMEM plus 10\% FBS and $1 \% \mathrm{~A} / \mathrm{B}$, and supplemented with $10 \mathrm{~mm} \beta$-glycerophosphate (Sigma, USA), $50 \mu \mathrm{g} / \mathrm{ml}$ ascorbic acid (Sigma, USA) and $10^{-8} \mathrm{M}$ dexamethasone (Sigma, USA) (Maniatopoulos et al., 1988) for $24 \mathrm{~h}$ before establishing the co-culture with monocytes/macrophages.

Human peripheral blood mononuclear cells (hPBMNCs) were isolated from buffy coats, following the differential centrifugation procedure using Histopaque 1077 described above. Monocytes/macrophages were obtained by cell adhesion selection (Bennett and Breit, 1994). Briefly, 50 000 hPBMNCs were plated in $6.5 \mathrm{~mm}$ Transwell ${ }^{\circledR}$ inserts with $0.4 \mu \mathrm{m}$ pore polycarbonate membrane (Corning, USA) and left to adhere for $24 \mathrm{~h}$ to select the monocyte/ macrophage subpopulation. After that time, non-adherent cells were discarded and the indirect contact co-cultures were set by placing the inserts in the 24-well culture plates wells with the hBMSCs seeded $24 \mathrm{~h}$ before. Both cell types were cultured in osteogenic medium for 2, 5 and 7 days. Anti-BMP-2 antibody (Abcam, UK) was added to the medium of half of the hBMSCs in co-culture at a dilution of 1:100 obtained after optimization (data not shown). Monocultures of hBMSCs in osteogenic medium were set as controls of the assay.

\subsection{Flow cytometry}

After isolation, hBMSCs at passage 1 were tested for the expression of CD45-FITC, CD73-PE and CD34-PE (all from BD Pharmingen, USA), CD31-APC (R\&D Systems, 
USA) and CD105-FITC and CD90-APC (eBiosciences, USA) markers. The cells were resuspended in cold PBS with $2 \%$ BSA and $100 \mu \mathrm{l}$ cell suspension with $2 \times 10^{5}$ cells, and incubated with the antibodies at the concentration advised by the manufacturer. After incubation for $20 \mathrm{~min}$ at room temperature in the dark, the cells were washed with PBS-BSA and resuspended in PBS with 1\% formaldehyde. Tubes were analysed in a BD FACScalibur flow cytometer (BD Biosciences, USA). Cells of interest were gated in a forward vs side-scatter dot-plot with a linear scale. Isotype controls were made to discern non-specific from specific staining. A minimum of 10000 gated events were acquired and displayed in dot-plots created using the Cyflogic software (version 1.2.1, CyFlo Ltd, Finland).

\section{3. dsDNA quantification}

Proliferation of hBMSCs was assessed after total dsDNA quantification along the culture time. The test was performed using the PicoGreen Quantification Kit (Invitrogen, CA, USA), according to the manufacturer's instructions, in a lysed cell suspension obtained after osmotic and thermal shocks. Fluorescence was read in a microplate reader (Bio-Tek, USA) at $485 \mathrm{ex} / 525 \mathrm{em}$.

\subsection{Alkaline phosphatase (ALP) activity quantification}

ALP activity quantification was performed in the same cell lysates used for the DNA quantification, obtained by osmotic and thermal shocks, and following an adapted p-nitrophenol assay (Maniatopoulos et al., 1988). Briefly, $20 \mu \mathrm{l}$ of lysate were incubated with $80 \mu \mathrm{l} p$-nitrophenol phosphate solution $(0.2 \% \mathrm{w} / \mathrm{w}$ in diethanolamine; Sigma, USA) at $37^{\circ} \mathrm{C}$ for $45 \mathrm{~min}$. The reaction was stopped using $80 \mu \mathrm{l} 2 \mathrm{M} \mathrm{NaOH}$ and $0.4 \mathrm{~mm}$ EDTA solution. The optical density of the samples was read at $405 \mathrm{~nm}$. A calibration curve was previously prepared using the $p$-nitrophenol standards (Sigma, USA) and used to extrapolate the ALP activity, which was then normalized against dsDNA results.

\subsection{Immunocytochemistry}

After $24 \mathrm{~h}$ of hPBMNCs culture, the polycarbonate membranes were cut off from the inserts and the selected monocytes/macrophages were stained for CD14-FITC monocytic lineage marker, using a mouse anti-human monoclonal antibody (BD Pharmingen, USA) in a concentration of $1: 15$. Non-specific binding was blocked by incubation with $3 \%$ bovine serum albumin (BSA) solution in PBS for $30 \mathrm{~min}$. After $1 \mathrm{~h}$ of incubation at room temperature, the cells were washed in PBS and counterstained with DAPI (Invitrogen) nuclear staining.

The presence of the stromal progenitor marker STRO-1 in the hBMSCs cultured for $24 \mathrm{~h}$ was screened using a mouse anti-human monoclonal antibody (Invitrogen), diluted 1:100. Briefly, after fixation with formalin, the cells were incubated with a 3\% BSA solution in PBS for 30 min to block non-specific binding. The cells were then incubated with the primary antibody for $1 \mathrm{~h}$ at room temperature and washed in PBS. AlexaFluor 488 secondary antibody (Invitrogen) was incubated with the cells for $1 \mathrm{~h}$ at room temperature. The cells were then washed in PBS and counterstained with DAPI (Invitrogen) nuclear staining.

The expression of CD14 and STRO-1 in the monocytes/ macrophages and hBMSCs was analysed using the Axioplan Imager Z1 fluorescence microscope (Zeiss, Germany).

\section{6. $P C R$ analysis}

\subsubsection{RNA extraction and CDNA production}

mRNA of hBMSCs was extracted, after each culture timepoint, using Tri-reagent (Sigma) according to the manufacturer's instructions. Briefly, $800 \mu$ TriZol were added per $1 \times 10^{6}$ cells and samples were homogenized by vigorous pipetting. Following $5 \mathrm{~min}$ incubation, $160 \mu \mathrm{l}$ chloroform (Sigma) were added to each sample; the samples were then incubated for $15 \mathrm{~min}$ at $4^{\circ} \mathrm{C}$ and centrifuged at $13000 \mathrm{rpm}$ at $4^{\circ} \mathrm{C}$ for $15 \mathrm{~min}$. After the centrifugation the aqueous part of each sample was collected and an equal volume of isopropanol (Sigma) was added. Following an overnight incubation at $-20^{\circ} \mathrm{C}$, the samples were centrifuged at $9000 \mathrm{rpm}$ at $4^{\circ} \mathrm{C}$ for $10 \mathrm{~min}$. The supernatants were discarded and the pellets were washed in ethanol, centrifuged at $9000 \mathrm{rpm}$ at $4{ }^{\circ} \mathrm{C}$ for $5 \mathrm{~min}$, and resuspended in $12 \mu \mathrm{l}$ RNase/DNase-free water (Gibco, UK). RNA quantity and purity were assessed using a NanoDrop ND-1000 spectrophotometer (NanoDrop Technologies, USA). Samples with a 260:280 ratio between 1.6 and 2.0 were used for cDNA synthesis. The cDNA synthesis was performed using iScript cDNA Synthesis kit (BioRad, USA) and the MiniOpticon Real-Time PCR Detection System (BioRad). An initial amount of $2 \mu \mathrm{g}$ mRNA was used in a total volume of $20 \mu \mathrm{l}$ RNase/DNase-free water.

\subsubsection{Quantitative real-time $P C R$}

Osteocalcin and osteopontin transcripts were quantified in the cDNA samples, using a quantitative real-time PCR reaction. For each sample, GAPDH was used as the housekeeping gene. The primers were designed using Primer 3 software (version 0.4.0) and synthesized by MWG Biotech (Germany) as follows: osteocalcin, forward 5'-GTG CAG AGT CCA GCA AAG GT-3', reverse 5'-TCC CAG CCA TTG ATA CAG GT-3'; osteopontin, forward 5'-CCC ACA GAC CCT TCC AAG TA5-3', reverse 5'-GGG GAC AAC TGG AGT GAA AA-3'; GAPDH, forward 5'-ACA GTC AGC CGC ATC TTC TT-3', reverse 5'-GAC AAG CTT CCC GTT CTC AG-3'. A concentration of $200 \mathrm{~nm}$ primer was used in a final volume of $25 \mu \mathrm{l}$ sample. No template controls were included in the study as controls. The real-time PCR 
reaction was done using the iQ Syber Green SuperMix (BioRad), following the manufacturer's instructions, in a MiniOpticon Real-Time PCR Detection System (BioRad).

The relative quantification of osteocalcin and osteopontin expression was performed using the $2^{-\Delta \mathrm{CT}}$ method (Perkin-Elmer User Bulletin No. 2). All values were first normalized against GAPDH values and then the coculture values against monoculture values.

\subsection{Statistical analysis}

Data were obtained from four separate experiments, with three replicates for each condition, and averaged. Standard deviation (SD) is reported as a measure of sample deviation. Statistical analysis was performed using Student's $t$-test for $n=4$ and values were considered statistically significant at $p \leq 0.05$. In the case of the quantitative real-time RT-PCR results, the statistical analysis was performed in ${ }^{\wedge} C_{T}$ values (Yuan et al., 2006).

\section{Results}

\subsection{Cell characterization}

In what concerns hBMSCs, cells were negative for CD45, CD34 and CD31. On the other hand, > 99\% of cells expressed the mesenchymal stem cell markers CD73, CD90 and CD105 (Figure 1).

The selected populations, monocytes/macrophages and hBMSCs were characterized $24 \mathrm{~h}$ after seeding, respectively, for the expression of CD14 (Figure 2A) and STRO-1 (Figure 2B) markers. The immunocytochemistry results showed that the cell population isolated from the bone marrow, albeit with different intensities between cells, was positive for STRO-1 mesenchymal stem cells. In addition, they also displayed a spindle-shaped morphology, as observed by optical microscopy (Figure 2C), typical of mesenchymal stem or progenitor stromal cells. The cells selected from the hPBMNCs were, as expected, positive for CD14.

\subsection{Proliferation of hBMSCs}

The effect of monocytes/macrophages on the proliferation of hBMSCs was assessed by quantifying the dsDNA of the hBMSCs cultures at different time points (Figure 3 ). The results indicated that hBMSCs cultured in the presence of monocytes/macrophages proliferated more than cells in the control conditions from day 2 onward $(p<0.02)$. This effect was eliminated when the co-cultures were established in the presence of the anti-BMP-2 antibody. For this condition, the proliferation rate of the hBMSCs was comparable to what was determined for the hBMSC monocultures.

\subsection{Osteogenic differentiation of hBMSCs}

The influence of monocytes/macrophages over the osteogenic differentiation of hBMSCs was assessed following different approaches: the quantification of the ALP activity, considered an early osteogenic marker, and the relative quantification of the osteopontin and osteocalcin transcripts.

The activity of the ALP enzyme on day 7 of culture was significantly higher $(p<0.02)$ in hBMSCs co-cultured with monocytes/macrophages than in hBMSC monocultures and in the co-cultures with monocytes/macrophages in the presence of anti-BMP-2 antibody. At day 5, however, the opposite was observed; the ALP activity in both
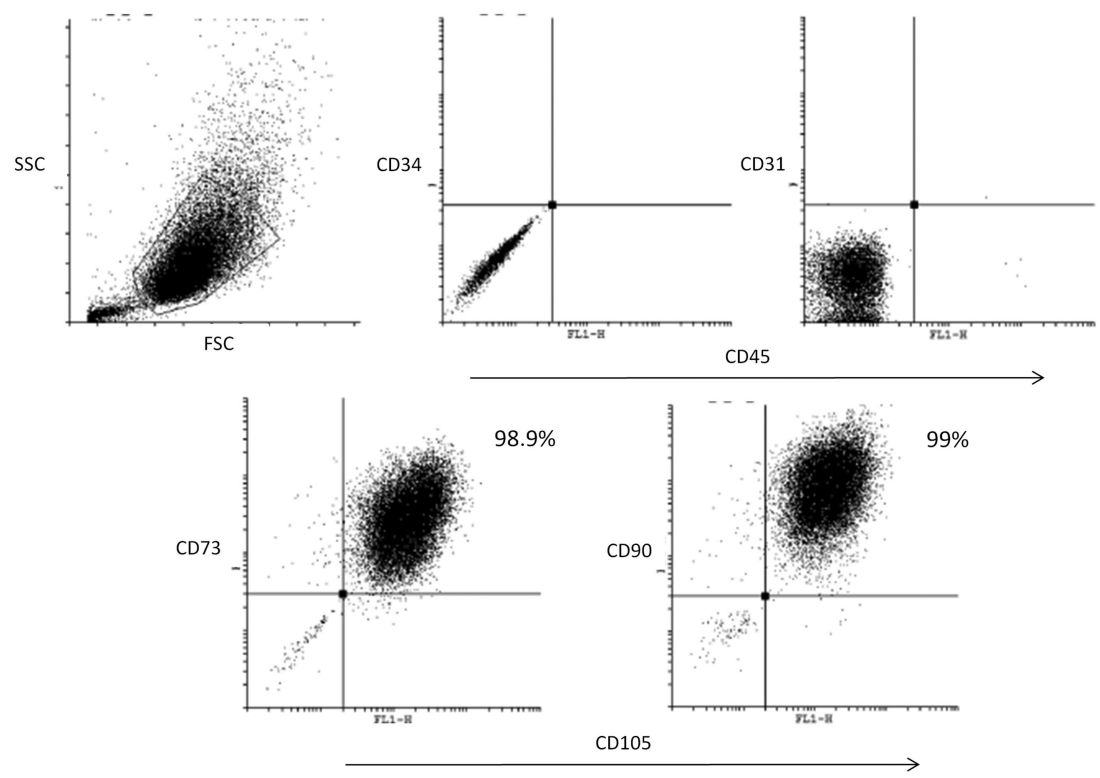

Figure 1. Flow cytometry analysis of hBMSCs at passage 1, just before seeding, confirming their progenitor phenotype 


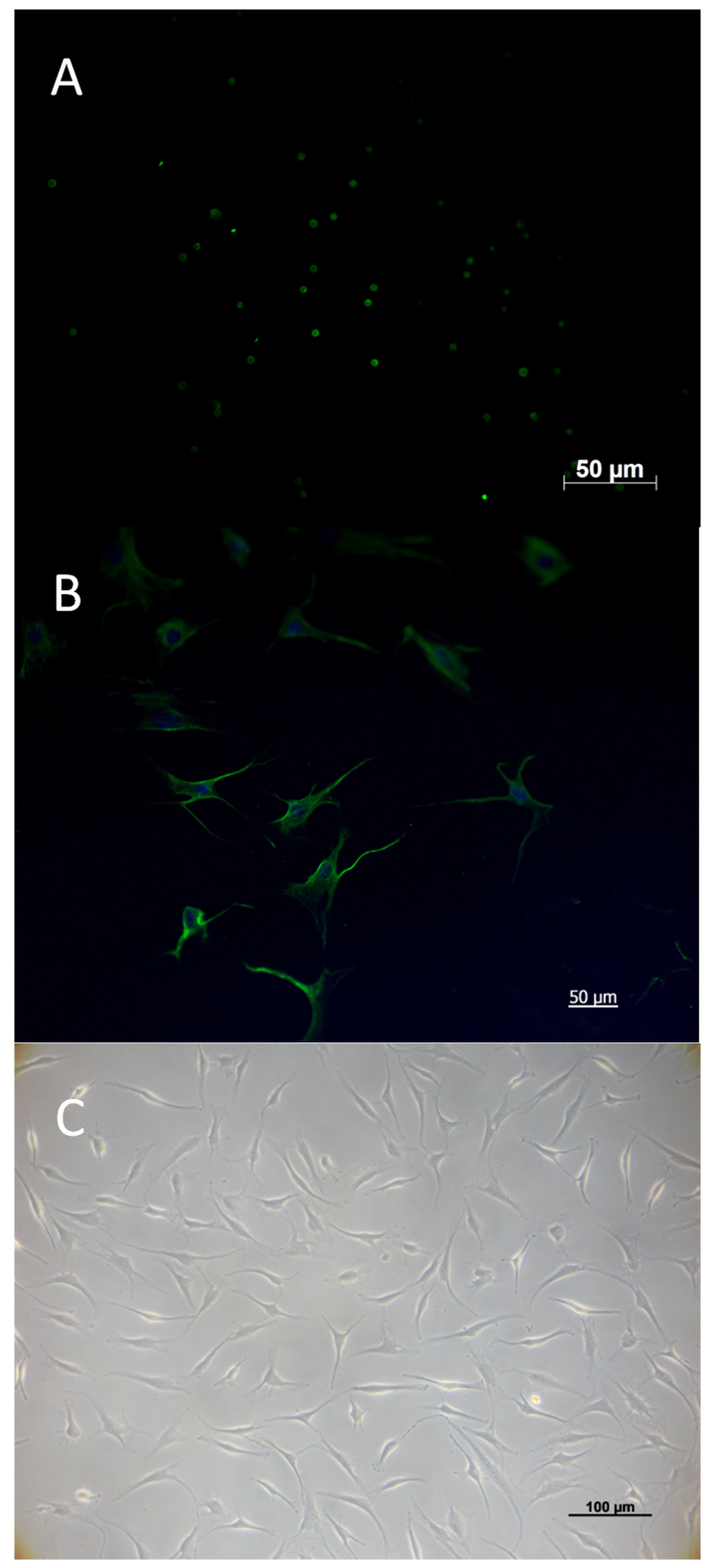

Figure 2. Characterization of monocytes/macrophage (A) and hBMSC (B,C) cultures after seeding. Monocyte/macrophage cultures were positive for CD14 (green; A). The hBMSCs expressed the stromal progenitor marker STRO-1 (green; B) and presented typical fibroblast-like morphology (C). Cell nuclei were counterstained with DAPI (blue; B)

hBMSC monocultures and co-cultures in the presence of anti-BMP-2 antibody had significantly higher $(p<0.02)$ values than in the co-cultures. Moreover, it is worth noting that the presence of anti-BMP-2 antibody in the culture at day 2 was not sufficient to reverse the increased ALP activity observed in the co-culture of hBMSCs and monocytes/macrophages. The ALP activity in the hBMSCs co-cultured with monocytes/macrophages in the presence of anti-BMP-2 antibody was significantly higher $(p<0.05)$ than in the hBMSCs in monoculture. (Figure 4).

The osteogenic differentiation of the hBMSCs was screened at the molecular level by quantifying the osteopontin and osteocalcin expression relative to
hBMSCs monocultures using real-time RT-PCR (Figure 5), the results of which showed that at days 2 and 7 of culture, the expression of osteocalcin in hBMSCs in cocultures had a fold change of approximately $4(p<0.02)$ in comparison to the monoculture condition. A similar trend was observed at day 5, although the fold change was around 2. In contrast, the expression of osteocalcin in hBMSCs co-cultured in the presence of anti-BMP-2 antibody was not different from what was observed for the hBMSCs monoculture (Figure 5A). Concerning the expression of osteopontin, an overexpression of this gene (fold change of 1.5) was observed in hBMSCs in co-cultures in comparison to hBMSCs in monoculture for all times of culture. Nonetheless, these observations were only statistically significant $(p<0.05)$ at days 2 and 7 . The presence of anti-BMP-2 antibody in the cultures annulled overexpression of the osteopontin gene, as demonstrated by the fold change of 1 when comparing co-cultures established in the presence of anti-BMP-2 antibody with hBMSCs in monoculture (Figure 5B).

\section{Discussion}

The study of cellular interactions after bone injury and during regeneration is of extreme importance for tissue engineering, in the sense that it can help researchers to better design engineered constructs and predict their outcome when applied in the clinics. After bone injury, monocytes are recruited to the injury site and differentiate into inflammatory macrophages, while in normal physiological conditions their recruitment to bone leads to their differentiation into bone-specific macrophages (Chang et al., 2008; Raggatt et al., 2009). Monocytes/ macrophages are also known to produce several osteogenic factors (Adams et al., 1985; Kreutz et al., 1993; O'Brien et al., 1994; Champagne et al., 2002; Takahashi et al., 2004) and several studies (Champagne et al., 2002; Chang et al., 2008) have suggested their strong influence over the osteogenic function or the differentiation of osteoblasts. Among those different osteogenic factors is BMP-2(Champagne et al., 2002; Chang et al., 2008).

Based on this knowledge, we hypothesized that peripheral blood monocytes/macrophages would also have a significant effect, in particular through BMP-2, over the osteogenic differentiation of hBMSCs, the most-studied cellular population, which has been proposed for the construction of bone tissue-engineering constructs. The magnitude of the monocyte/macrophage effect was further addressed by determining whether BMP-2 and the typical dexamethasone-containing osteogenic medium would have a synergistic effect, over a short period of time, on the osteogenic differentiation of hBMSCs.

In order to guarantee the validity of the work, our first concern was to use a progenitor cell population from the bone marrow, intimately linked with bone tissue. More than $95 \%$ of the isolated hBMSCs expressed CD73, CD90 and CD105 and were negative to CD14, CD34 and CD31 $(<2 \%)$, conforming to the accepted mesenchymal 


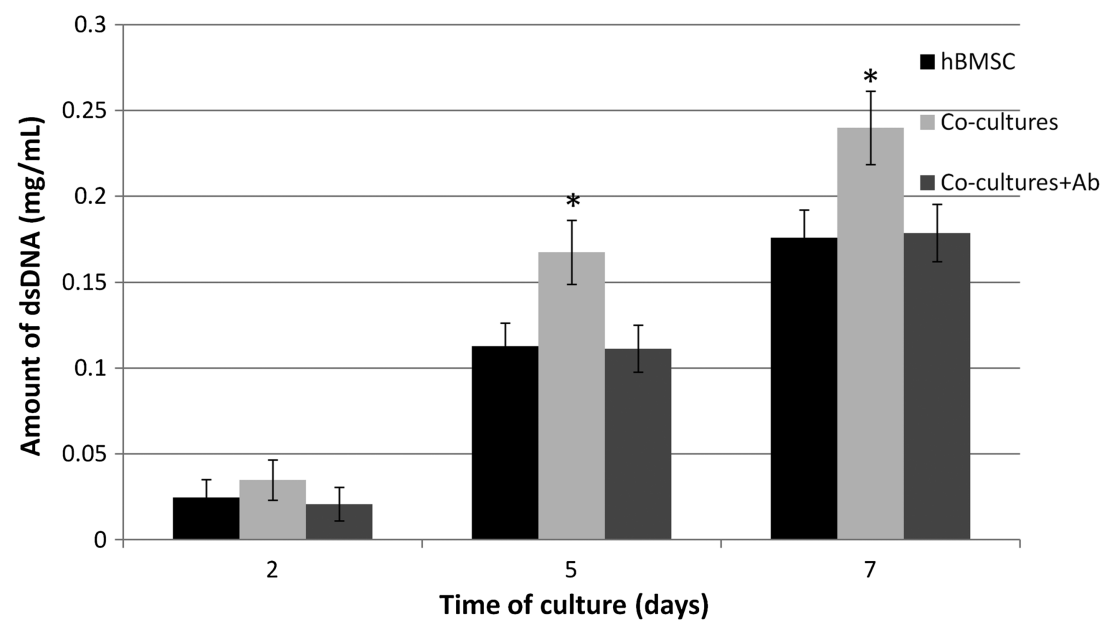

Figure 3. Amount of dsDNA that correlates with cell number quantified along culture in hBMSCs and in co-culture with monocytes/ macrophages with (co-cultures $+\mathrm{Ab}$ ) and without (co-cultures) anti-BMP-2 antibody; ${ }^{*} p<0.02$, relating to hBMSC cultures for the same time point

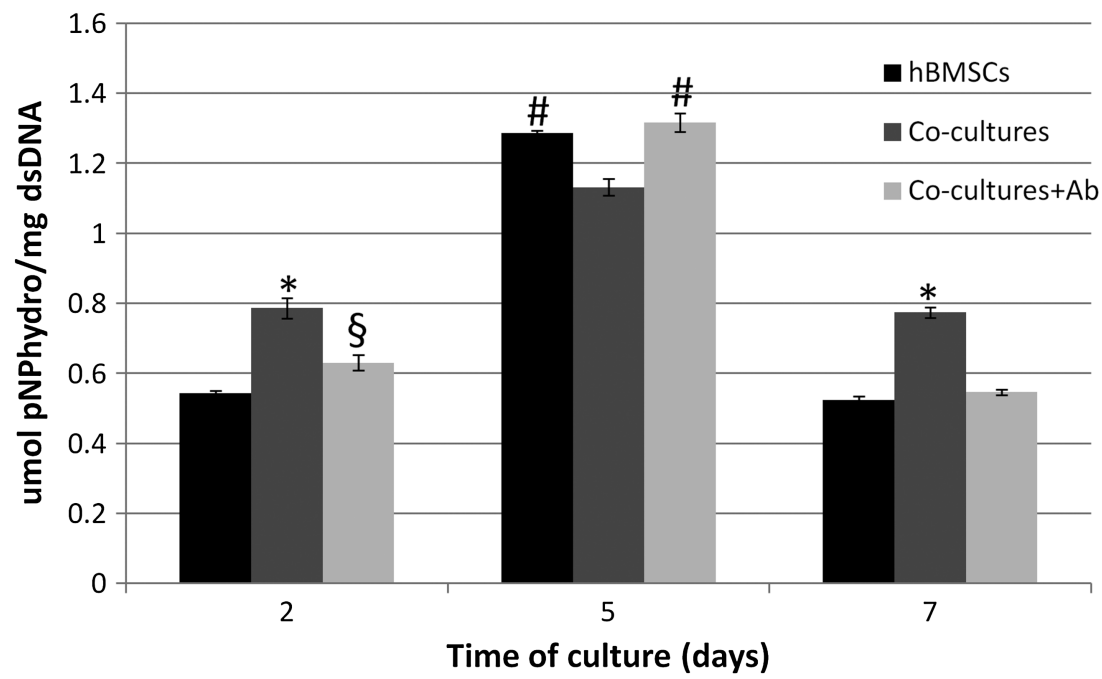

Figure 4. Amount of hydrolysed p-nitrophenol phosphate that correlates with the ALP activity quantified along culture in hBMSCs and in co-culture with monocytes/macrophages, with (co-cultures $+\mathrm{Ab}$ ) and without (co-cultures) anti-BMP-2 antibody. Results were normalized against dsDNA values. ${ }^{\S} p<0.05$ and ${ }^{*} p<0.02$, relating to hBMSCs for the same time point; \#p $<0.02$, relating to cocultures for the same time point

stem cell marker pattern. After seeding cells in the cell culture plate wells, positivity for STRO-1 marker and spindle-shape morphology confirmed that the cells maintained their mesenchymal phenotype (Dominici et al., 2006). The confirmation of the monocyte/macrophage lineage of the cells co-cultured with the hBMSCs was demonstrated by the expression of CD14 in the cells selected by adherence. The different intensities of expression observed after immunocytochemistry reflected the different expression pattern of CD14 in monocytes and macrophages (Zannettino et al., 2007).

Cell proliferation results showed that the presence of monocytes/macrophages had a positive effect on hBMSC proliferation and that this effect was inhibited by adding anti-BMP-2 antibody. Real-time RT-PCR demonstrated the expression of BMP-2 by monocytes/macrophages (results not shown). This suggests that BMP-2 produced by monocytes/macrophages is involved in promoting hBMSCs proliferation. Reports in the literature concerning the effect of BMP-2 on bone marrow stromal cells proliferation are contradictory (Fromigué et al., 1998; Gori et al., 1999; Lou et al., 1999), which indicates that this effect may be highly dependent on the type of culture model used. Some studies using hBMSCs showed a diminished proliferation when the cells were cultured in the presence of BMP-2 (Lecanda et al., 1998; Francesca et al., 1999). It is also well understood how cell proliferation and differentiation are inversely regulated (Stein and Lian, 1993). This seems to indicate that, in our model, BMP-2 acts synergistically with other soluble factors in the culture, resulting in proliferation of hBMSCs.

ALP activity in the co-cultures was inhibited to the level of monocultures at days 2 and 7 of culture after BMP-2 blocking, which reveals the direct effect of BMP-2 over ALP activity, as published by others (Lecanda et al., 1998). The fact that for day 5 the opposite result was verified could be an artifact of the experiment related to the culture model used. This is further reinforced by the 

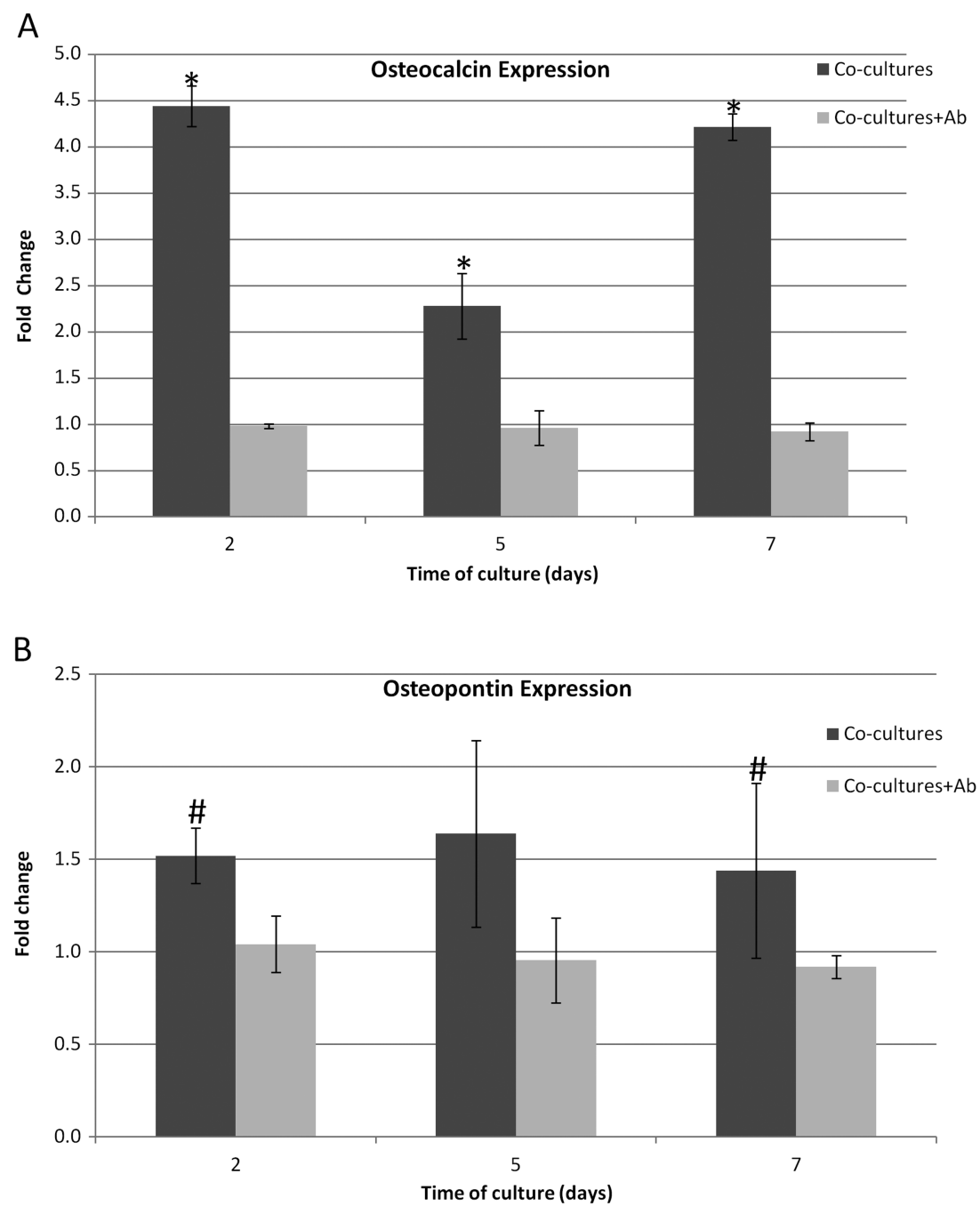

Figure 5. Osteocalcin (A) and osteopontin (B) relative expressions calculated after quantitative real-time RT-PCR. The results were first normalized against GAPDH and then against hBMSC cultures. ${ }^{*} p<0.02$ and ${ }^{\#} p<0.05$, statistically different ${ }^{\triangle} \mathrm{C}_{\mathrm{T}}$ values in comparison with hBMSCs cultures at the same time point

similar trend observed for osteopontin expression. Nevertheless, analysis of the expression of osteocalcin and osteopontin markers confirmed that the overexpression of those markers in the presence of monocytes/macrophages was eliminated by blocking BMP-2. Once again, this corresponds to what is described as the effect of BMP-2 over the expression of osteogenic markers in hBMSCs (Lecanda et al., 1998). Osteocalcin and osteopontin are associated with the osteogenic differentiation and mineralization processes of hBMSCs (Fromigué et al., 1998; Lecanda et al., 1998; Gori et al., 1999), thus an overexpression of these markers was expected.

It is also known that hBMSCs produce BMP-2 during osteogenic differentiation (Frank et al., 2002). In the present study, the control condition, hBMSCs monocultures in osteogenic medium, clearly demonstrates how significant the presence of monocytes/macrophages is. In addition, the effect of monocyte/macrophage-produced BMP-2 was evaluated while cells were already being directed to the osteogenic lineage by dexamethasone-containing osteogenic medium. Therefore, a synergistic effect, to our knowledge unreported so far, of BMP-2 and the osteogenic factors in the culture medium cannot be discarded. Both BMP-2 (Nishimura et al., 2002; Lian and Stein, 2003) and dexamethasone (Phillips et al., 2006; Mikami et al., 2007) ultimately upregulate a cascade of osteoblastic transcription factors, among which is Runx2, although involving two different molecular pathways. In the case of dexamethasone, it has been proposed that its osteogenic effect is accomplished through a glucocorticoid receptor-mediated mechanism that modulates Runx2 phosphorylation via mitogen-activated protein kinase phosphatase-1 (MKP-1) (Phillips et al., 2006). On the other hand, it is well established that BMP-2 upregulates Runx2 via the Smad pathway (Schmitt et al., 1999a, 1999b). Therefore, it is possible that the mechanisms triggered by both dexamethasone and BMP-2 have a cumulative effect on the osteogenic differentiation of hBMSCs. The mechanisms orchestrating the osteogenic differentiation of hBMSCs are as complex as important to address, even more if taking in account a possible synergistic effect of two known osteogenic factors. It is thus our consideration that the mechanisms proposed here should be the subject of a more pervasive study. 


\section{Conclusions}

The present report confirms that peripheral blood monocytes/macrophages are capable of deeply influencing hBMSC metabolism. It is also strongly suggested that this mechanism is regulated by BMP-2 production by monocytes/macrophages. The effect of BMP-2 on hBMSC differentiation was cumulative to that exerted by dexamethasone-containing osteogenic medium and, additionally, promoted hBMSC proliferation. Synergistic effects between dexamethasone- and BMP-2-induced effects may explain this result, but a more profound analysis, in both molecular and experimental time-spanning terms, is needed.

\section{References}

Adams JS, Singer FR, Gacad MA et al. 1985; Isolation and structural identification of 1,25-dihydroxyvitamin D3 produced by cultured alveolar macrophages in sarcoidosis. J Clin Endocrinol Metab 60(5): 960.

Aggarwal S, Pittenger MF. 2005; Human mesenchymal stem cells modulate allogeneic immune cell responses. Blood 105 (4): 1815-1822.

Bennett S, Breit S. 1994; Variables in the isolation and culture of human monocytes that are of particular relevance to studies of HIV. J Leukocyte Biol 56(3): 236-240.

Bessa PC, Casal M, Reis RL. 2008; Bone morphogenetic proteins in tissue engineering: the road from the laboratory to the clinic, part I (basic concepts). J Tissue Eng Regen Med 2(1): 1-13.

Champagne CM, Takebe J, Offenbacher S et al. 2002; Macrophage cell lines produce osteoinductive signals that include bone morphogenetic protein-2. Bone $30(1)$ : 26-31.

Chang MK, Raggatt LJ, Alexander KA et al. 2008; Osteal tissue macrophages are intercalated throughout human and mouse bone lining tissues and regulate osteoblast function in vitro and in vivo. $J$ Immunol 181(2): 1232.

Dimitriou R, Tsiridis E, Giannoudis PV. 2005; Current concepts of molecular aspects of bone healing. Injury 36(12): 1392-1404.

Dominici M, Le Blanc K, Mueller I et al. 2006; Minimal criteria for defining multipotent mesenchymal stromal cells. International Society for Cellular Therapy position statement. Cytotherapy 8(4): 315-317.

Einhorn TA. 1998; The cell and molecular biology of fracture healing. Clin Orthop Rel Res 355: S7.

Francesca G, Thierry T, Kevin CH et al. 1999; Differentiation of human marrow stromal precursor cells: bone morphogenetic protein-2 increases OSF2/CBFA1, enhances osteoblast commitment, and inhibits late adipocyte maturation. J Bone Miner Res 14(9): 1522-1535.

Frank O, Heim M, Jakob M et al. 2002; Realtime quantitative RT-PCR analysis of human bone marrow stromal cells during osteogenic differentiation in vitro. $J$ Cell Biochem 85(4): 737-746.

Fromigué O, Marie PJ, Lomri A. 1998; Bone morphogenetic protein-2 and transforming growth factor- $\beta 2$ interact to modulate human bone marrow stromal cell proliferation and differentiation. $J$ Cell Biochem 68(4): 411-426.

Fujikawa Y, Sabokbar A, Neale S et al. 1996; Human osteoclast formation and bone resorption by monocytes and synovial macrophages in rheumatoid arthritis. $\mathrm{Br}$ Med $J$ 55(11): 816.

Gori F, Thomas T, Hicok KC et al. 1999; Differentiation of human marrow stromal precursor cells: bone morphogenetic protein-2 increases OSF2/CBFA1, enhances osteoblast commitment, and inhibits late adipocyte maturation. J Bone Miner Res 14(9): 1522-1535.

Haynes DR, Crotti TN, Loric M et al. 2001; Osteoprotegerin and receptor activator of nuclear factor $\kappa \mathrm{B}$ ligand (RANKL) regulate osteoclast formation by cells in the human rheumatoid arthritic joint. Rheumatology 40(6): 623

Hume DA, Ross IL, Himes SR et al. 2002; The mononuclear phagocyte system revisited. $J$ Leukocyte Biol 72(4): 621.

Jiang XX, Zhang Y, Liu B et al. 2005; Human mesenchymal stem cells inhibit differentiation and function of monocyte-derived dendritic cells. Blood 105(10): 4120-4126.

Kreutz M, Andreesen R, Krause SW et al. 1993; 1,25-dihydroxyvitamin D3 production and vitamin D3 receptor expression are developmentally regulated during differentiation of human monocytes into macrophages. Blood 82(4): 1300

Lecanda F, Avioli LV, Cheng SL. 1998; Regulation of bone matrix protein expression and induction of differentiation of human osteoblasts and human bone marrow stromal cells by bone morphogenetic protein2. J Cell Biochem 67(3): 386-398.

Lian JB, Stein GS. 2003; Runx2/Cbfa1: a multifunctional regulator of bone formation. Curr Pharmaceut Design 9(32): 2677-2685.

Linkhart TA, Mohan S, Baylink DJ. 1996; Growth factors for bone growth and repair: IGF, TGF $\beta$ and BMP. Bone 19(1S1): $1-12$.

Lou J, Xu F, Merkel K et al. 1999; Gene therapy: adenovirus-mediated human bone morphogenetic protein-2 gene transfer induces mesenchymal progenitor cell proliferation and differentiation in vitro and bone formation in vivo. $J$ Orthop Res 17(1) 43-50.

Maniatopoulos C, Sodek J, Melcher AH. 1988; Bone formation in vitro by stromal cells obtained from bone marrow of young adult rats. Cell Tissue Res 254(2): 317-330.

Mikami Y, Omoteyama K, Kato S et al. 2007; Inductive effects of dexamethasone on the mineralization and the osteoblastic gene expressions in mature osteoblast-like ROS17/2.8 cells. Biochem Biophys Res Commun 362(2): 368-373.

Mundy GR. 1993; Hormonal factors which regulate bone resorption. Handbook Exp Pharmacol 107: 215-247.
Nishimura R, Hata $\mathrm{K}$, Harris SE et al. 2002; Core-binding factor $\alpha 1$ (Cbfa1) induces osteoblastic differentiation of C2C12 cells without interactions with Smad1 and Smad5. Bone 31(2): 303-312.

O'Brien ER, Garvin MR, Stewart DK et al. 1994; Osteopontin is synthesized by macrophage, smooth muscle, and endothelial cells in primary and restenotic human coronary atherosclerotic plaques. Arterioscler Thromb Vasc Biol 14(10): 1648.

Parfitt AM. 1982; The coupling of bone formation to bone resorption: a critical analysis of the concept and of its relevance to the pathogenesis of osteoporosis. Metab Bone Dis Relat Res 4(1): 1-6.

Phillips JE, Gersbach CA, Wojtowicz AM et al. 2006; Glucocorticoid-induced osteogenesis is negatively regulated by Runx2/Cbfa1 serine phosphorylation. $J$ Cell Science 119 (3): 581.

Pirraco RP, Marques AP, Reis RL. 2009; Cell interactions in bone tissue engineering. $J$ Cell Mol Med 14(1-2): 93-102.

Quinn JMW. 1998; Human osteoclast formation from blood monocytes, peritoneal macrophages, and bone marrow cells. Calc Tissue Int 62(6): 527-531.

Raggatt L, Chang M, Alexander Ket al. 2009; Osteomacs: osteoclast precursors during inflammatory bone disease but regulators of physiologic bone remodeling. Bone 44: S136-137.

Rodan GA, Martin TJ. 1981; Role of osteoblasts in hormonal control of bone resorption - a hypothesis. Calc Tissue Int 33(1): 349-351.

Ryan JM, Barry FP, Murphy JM et al. 2005; Mesenchymal stem cells avoid allogeneic rejection. $J$ Inflamm 2(1): 8.

Sasaki T, Hong MH, Udagawa N et al. 1994; Expression of vacuolar $\mathrm{H}^{+}$-ATPase in osteoclasts and its role in resorption. Cell Tissue Res 278(2): 265-271.

Schmitt JM, Hwang K, Winn SR et al. 1999a; Bone morphogenetic proteins: an update on basic biology and clinical relevance. $J$ Orthop Res 17(2): 269-278.

Schmitt JM, Hwang K, Winn SR et al. 1999b; Bone morphogenetic proteins: an update on basic biology and clinical relevance. $J$ Orthop Res 17(2): 269.

Stein GS, Lian JB. 1993; Molecular mechanisms mediating proliferation/differentiation interrelationships during progressive development of the osteoblast phenotype. Endocr Rev 14(4): 424-442.

Takahashi F, Takahashi K, Shimizu K et al. 2004; Osteopontin is strongly expressed by alveolar macrophages in the lungs of 
acute respiratory distress syndrome. Lung 182(3): 173-185.

Teitelbaum SL, Abu-Amer Y, Ross FP. 1995; Molecular mechanisms of bone resorption. $J$ Cell Biochem 59(1): 1-10.

Teitelbaum SL, Tondravi MM, Ross FP. 1997; Osteoclasts, macrophages, and the molecular mechanisms of bone resorption. J Leukocyte Biol 61(4): 381-388.

Tintut Y, Patel J, Territo M et al. 2002; Monocyte/macrophage regulation of vascular calcification in vitro. Am Heart Assoc 105: 650-655.

Udagawa N, Takahashi N, Akatsu T et al. 1990; Origin of osteoclasts: mature monocytes and macrophages are capable of differentiating into osteoclasts under a suitable microenvironment prepared by bone marrow-derived stromal cells. Proc Natl Acad Sci USA 87(18): 7260.

Valentin-Opran A, Wozney J, Csimma C et al. 2002; Clinical evaluation of recombinant human bone morphogenetic protein-2. Clin Orthop Rel Res 395: 110-120.

Yuan JS, Reed A, Chen F et al. 2006; Statistical analysis of real-time PCR data. BMC Bioinform 7(1): 85 .

Zannettino ACW, Paton S, Kortesidis A et al. 2007; Human mulipotential mesenchy$\mathrm{mal} /$ stromal stem cells are derived from a discrete subpopulation of STRO1bright/CD34/CD45/glycophorin-A-bone marrow cells. Haematologica 92(12): 1707-1708. 